\title{
Estrogen receptor beta as a prognostic factor in breast cancer patients: A systematic review and meta-analysis
}

\author{
Weige Tan ${ }^{1,2 *}$, Qian Li ${ }^{1,2 *}$, Kai Chen ${ }^{1,2}$, Fengxi Su1,2, Erwei Song ${ }^{1,2,3}$ and Chang Gong ${ }^{1,2}$ \\ ${ }^{1}$ Guangdong Provincial Key Laboratory of Malignant Tumor Epigenetics and Gene Regulation, Sun Yat-Sen Memorial Hospital, \\ Sun Yat-Sen University, Guangzhou, China \\ 2 Breast Tumor Center, Sun Yat-Sen Memorial Hospital, Sun Yat-Sen University, Guangzhou, China \\ ${ }^{3}$ Collaborative Innovation Center for Cancer Medicine, Guangzhou, China \\ * These authors have contributed equally to this work \\ Correspondence to: Chang Gong, email: changgong282@163.com
}

Erwei Song, email: songerwei02@aliyun.com

Keywords: estrogen receptor beta, breast cancer, survival, endocrine therapy, prognostic factor

Received: October 11,2015 Accepted: January 24, $2016 \quad$ Published: February 06, 2016

\section{ABSTRACT}

Background: The prognostic role of estrogen receptor beta (ERß) in early-stage breast cancer is unclear. We performed a systematic review and meta-analysis to evaluate the prognostic value of ER $\beta$ in early-stage breast cancer patients.

Method: We searched Medline, Embase, and the Web of Science for studies published between 1990 and 2015 that assessed ER $\beta$ status in breast cancer patients. A total of 25 studies comprising 9919 patients fitting our inclusion and exclusion criteria were included. The hazard ratios of ER $\beta$ status were extracted for diseases free survival (DFS)/ ) and overall survival (OS). Random or fixed-effects models were used when appropriate, and between-study heterogeneity was assessed.

Results: In the 20 studies that assessed ER $\beta$ status using immunohistochemical (IHC) methods, we observed significantly improved DFS in patients positive for ER $\beta$ $1(\mathrm{HR}=0.56,95 \% \mathrm{CI} 0.40-0.78, P=0.0007)$ and $\mathrm{ER} \beta-2$ (HR=0.67, 95\%CI 0.45-1.00, $P=0.05)$. Improved OS was associated with a positive status for pan-ER $\beta(H R=0.60$, $95 \% \mathrm{CI} 0.45-0.80, P=0.0004)$ and ER $\beta-2$ (HR=0.44, 95\%CI 0.31-0.62, $P<0.0001)$. In ERa-positive patients, ER $\beta$ positivity was not associated with DFS (HR=0.77, 95\%CI 0.46-1.27, $P=0.31)$ or OS (HR=0.64, 95\%CI 0.37-1.11, $P=0.11)$. In contrast, ER $\beta$ expression was significantly associated with increased DFS (HR $=0.37,95 \%$ CI $0.14-$ $0.93, P=0.03)$ or OS (HR=0.44, 95\%CI 0.30-0.65, $P<0.0001)$ in ERa-negative patients. We did not observe an association between ER $\beta$ mRNA levels and DFS and OS.

Conclusion: In this study, we showed that IHC ERß status, rather than mRNA levels, is a prognostic factor that is associated with DFS and OS in breast cancer patients. The prognostic value of ERß may be higher in ERa-negative patients than in ERa-positive patients.

\section{INTRODUCTION}

Estrogen receptor $\alpha(\mathrm{ER} \alpha)$ has been established as a significant predictor of the response to endocrine therapy in breast cancer patients. Immunohistochemical (IHC) examination of ER $\alpha$ status is the standard-of-care pathological evaluation used to guide adjuvant endocrine therapy after surgery. Anti-estrogen approaches are recommended in ER $\alpha+$ patients. The discovery of a second $E R, E R \beta$, has lead to the re-evaluation of estrogen activity in normal mammary development, breast tumorigenesis and tumor progression. Despite over 15 years of research on ER $\beta$, its clinical significance remains unclear. Mann et al. [1] were the first to report the significance of ER $\beta$ in predicting long-term clinical outcomes (e.g., disease-free survival) in breast cancer patients, a result confirmed by other studies [2-4]. However, conflicting findings suggest that ER $\beta$ status is not associated with survival $[5,6]$. The aim of the present systematic review and meta-analysis was to investigate the association of $\operatorname{ER} \beta$ status (positive vs. negative) and long-term clinical outcomes (e.g. disease-free survival, overall survival) of breast cancer patients. 


\begin{tabular}{|c|c|c|c|c|c|c|c|c|c|}
\hline \multicolumn{10}{|c|}{ Table 1a: Features of included studies. } \\
\hline \multirow{2}{*}{ References } & \multirow{2}{*}{ Year } & \multirow{2}{*}{ Patients (n) } & \multirow{2}{*}{$\begin{array}{l}\text { Mean } \\
\text { age }\end{array}$} & \multirow{2}{*}{ Methods • } & \multirow{2}{*}{$\begin{array}{l}\text { ERb } \\
\text { assessment •• }\end{array}$} & \multicolumn{2}{|c|}{ ERb status } & \multirow{2}{*}{$\begin{array}{l}\text { Median Follow } \\
\text { up(Months) }\end{array}$} & \multirow{2}{*}{ Quality Score } \\
\hline & & & & & & ERb+ & ERb- & & \\
\hline \multirow{3}{*}{ Borgquist et al.[11] } & \multirow{3}{*}{2008} & 512 & \multirow{3}{*}{64.2} & \multirow{3}{*}{ i } & \multirow{3}{*}{ ii } & 167 & 312 & 106 & \multirow{3}{*}{$* * * * *$} \\
\hline & & 114 \# & & & & 60 & 54 & NA & \\
\hline & & 139 \#\# & & & & 71 & 68 & NA & \\
\hline Chantzi et al.[20] & 2013 & 95 & 52 & i & i & $\begin{array}{l}\mathrm{b} 1: 66 \\
\mathrm{~b} 2: 65\end{array}$ & $\begin{array}{l}\mathrm{b} 1: 29 \\
\mathrm{~b} 2: 30\end{array}$ & NA & $* * * * * *$ \\
\hline $\begin{array}{l}\text { Gruvberger-Saal } \\
\text { et al.[5]\$, ๆศศๆ }\end{array}$ & 2007 & 425 & NA & i & ii & 262 & 91 & 174 & $* * * * * * *$ \\
\hline 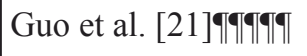 & 2014 & 490 & 49 & i & ii & 110 & 380 & 60 & $* * * * * * *$ \\
\hline Honma et al. [2]§ & 2008 & 442 & 56 & i & ii & 405 & 37 & 133 & $* * * * * *$ \\
\hline \multirow{3}{*}{ Hopp et al.[12] } & \multirow{3}{*}{2004} & 305 & \multirow{3}{*}{62} & \multirow{3}{*}{ iii } & \multirow{3}{*}{$\mathrm{v}$} & 141 & 164 & 65 & \multirow{3}{*}{$* * * * *$} \\
\hline & & $186 \#$ & & & & 89 & 97 & 74 & \\
\hline & & $119 \# \#$ & & & & 52 & 67 & 50 & \\
\hline Kim et al.[13] & 2012 & 139 & NA & ii & iii & 53 & 87 & 48 & $* * * * *$ \\
\hline Mahle et al.[14]§ & 2009 & 145 & 63 & i & ii & 129 & 16 & 165 & $* * * * * * *$ \\
\hline \multirow{2}{*}{ Mann et al.[1] } & \multirow{2}{*}{2001} & 47 \#\# & NA & \multirow{2}{*}{$\mathrm{i}$} & ii & 33 & 14 & 88 & \multirow{2}{*}{$* * * *$} \\
\hline & & 118 \# & NA & & ii & 78 & 40 & 49 & \\
\hline Markey et al.[28] & 2009 & 121 & 54 & ii & iii & 50 & 71 & 38 & $* * *$ \\
\hline Myers et al.[15] & 2004 & 150 & NA & i & $\mathrm{i}$ & 87 & 63 & 27 & $* * *$ \\
\hline Nakopoulou et al.[3] & 2004 & 181 & 61 & i & ii & 128 & 50 & 76 & $* * * * *$ \\
\hline Novelli et al.[6] & 2008 & 936 & NA & i & ii & 520 & 416 & 50 & $* * * * * * *$ \\
\hline Omoto et al.[18] & 2002 & 57 & 60.9 & $\mathrm{i}$ & ii & 15 & 42 & 48 & $* * *$ \\
\hline Omoto et al.[17] & 2001 & 88 & $54 \&$ & i & $\mathrm{i}$ & 52 & 36 & NA & $* * * *$ \\
\hline \multirow{2}{*}{ O'Neill et al. $9[16]$} & \multirow{2}{*}{2004} & & NA & i & ii & 117 & 10 & & \\
\hline & & $10 /$ & NA & ii & iii & 86 & 35 & INA & in \\
\hline Palmieri et al.[19] & 2004 & 82 & 59 & i & i & 33 & 46 & 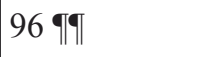 & $* * * *$ \\
\hline Qui et al.[22] & 2009 & 308 & 58 & i & ii & 123 & 185 & 48 & $* * *$ \\
\hline Shaaban et al.[23] & 2008 & 880 & NA & i & i, ii & 558 & 112 & 94 & $* * * * * *$ \\
\hline & & & & i & $\mathrm{i}$ & 103 & 47 & & \\
\hline 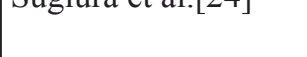 & 2007 & 100 & DJ & ii & iii & 52 & 98 & & \\
\hline & & 141 & & i & i & 100 & 41 & & \\
\hline$[4] \rrbracket, \S$ & 2007 & 100 & 68 & ii & iii & 34 & 30 & BCR:79 & Pans \\
\hline
\end{tabular}




\begin{tabular}{|c|c|c|c|c|c|c|c|c|c|}
\hline Wen et al.[25] & 2002 & 116 & 53.7 & iii & $\mathrm{v}$ & 40 & 76 & 35.3 & $* * * * * *$ \\
\hline \multirow{4}{*}{ Wimberly et al.[26] } & \multirow{4}{*}{2014} & Yale-1:649 & \multirow{4}{*}{ NA } & \multirow{4}{*}{ iv } & \multirow{4}{*}{ iv } & $\begin{array}{l}\text { b1:228 } \\
\text { b5:209 }\end{array}$ & $\begin{array}{l}\text { b1:228 } \\
\text { b5:209 }\end{array}$ & 95 & $* * * *$ \\
\hline & & Yale-2:398 & & & & $\begin{array}{l}\text { b1:147 } \\
\text { b5:153 }\end{array}$ & $\begin{array}{l}\text { b1:148 } \\
\text { b5:152 }\end{array}$ & 123 & $* * * *$ \\
\hline & & $\begin{array}{l}\text { Toronto: } \\
976\end{array}$ & & & & \begin{tabular}{|l|} 
b1:225 \\
b5:153
\end{tabular} & $\begin{array}{l}\text { b1:225 } \\
\text { b5:153 }\end{array}$ & 98.2 & $* * * *$ \\
\hline & & $\begin{array}{l}\text { NCI-PBCS: } \\
1375\end{array}$ & & & & b5:467 & b5:468 & 116 & $* * * *$ \\
\hline Yan et al. $\| \uparrow ף, \S[27]$ & 2011 & 147 & NA & i & ii & 90 & 20 & 64 & $* * *$ \\
\hline Zhang et al.[29] & 2014 & 279 & 48.8 & i & ii & 40 & 109 & 92 & $* * *$ \\
\hline \multicolumn{10}{|c|}{ 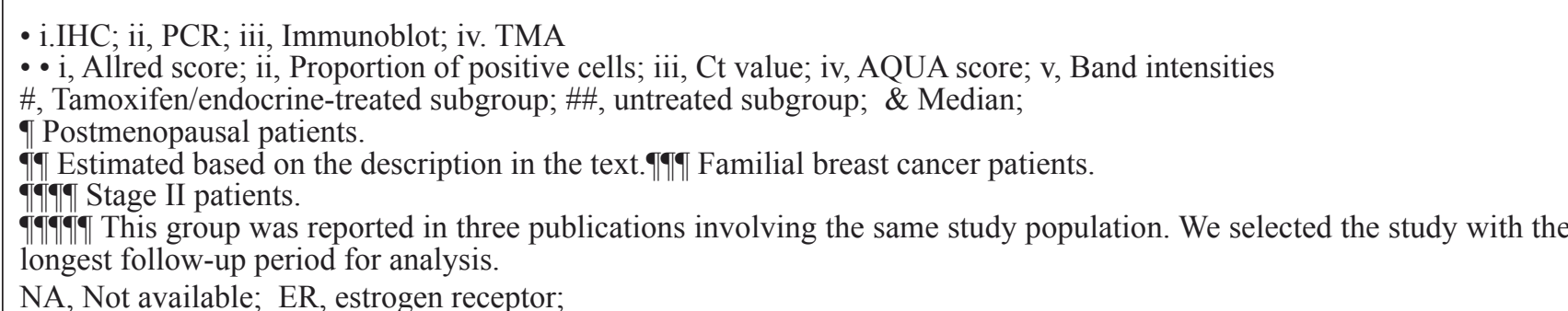 } \\
\hline
\end{tabular}

\section{RESULTS}

\section{Study characteristics}

Twenty-five studies [1-25] with the full text available were identified and included in this study (Figure 1). We examined the reference list of each study and did not identify any further studies for inclusion in our analysis. We included a total of 9919 patients from these studies. All publications were full-text articles. The features of the included studies are summarized in Table 1a, 1b. The mean patient age ranged from 48 to 68 years, and the median follow-up ranged from 27 to 174 months. Nine of the included studies had a quality score $\geq 6$. None of the included studies were prospective, randomized trials. All of the studies were retrospective and did not report any information about allocation concealment or blinding methods. The matching criteria varied among the studies. Most of the studies reported the length of the follow-up period, and 12 of them exhibited a sufficiently long follow-up (defined as a median follow-up time $>60$ months) for the outcomes to be determined. The treatment of missing data was not sufficiently described in most of the studies.

\section{The effect of ERß on DFS}

A total of 16 studies[2-7, 11-17, 19,25] with available DFS used IHC as the method of ER $\beta$ assessment.
Pooling the data showed that a positive status for ER $\beta$ 1 (HR $=0.56,95 \% \mathrm{CI} 0.40-0.78, P=0.0007$; heterogeneity: $\left.P<0.01, \mathrm{I}^{\wedge} 2=64 \%\right)$ or ER $\beta-2 \quad(\mathrm{HR}=0.67,95 \% \mathrm{CI} 0.45$ 1.00, $P=0.005$; heterogeneity: $P=0.10, \mathrm{I}^{\wedge} 2=45 \%$ ) was significantly associated with improved DFS (Figure 2). Two studies $[8,21]$ used immunoblotting to assess panER $\beta$ status. Pooling the data revealed that a positive pan-ER $\beta$ status was associated with an improved DFS ( $\mathrm{HR}=0.51,95 \% \mathrm{CI} 0.35-0.75, P=0.0007$; heterogeneity: $P=0.33, \mathrm{I}^{\wedge} 2=9 \%$; Figure $\left.\mathrm{S} 1\right)$. Five studies $[4,9,12,20,24]$ assessed ER $\beta$ mRNA levels via PCR, and no association between total ER $\beta$ mRNA levels and DFS was detected (Figure S2). Wimberly et al. [22] employed a tissue microarray (TMA) to assess the pan-ER $\beta$ and ER $\beta-1$ statuses of four independent populations. However, there was no association between ER $\beta$ status and DFS in these populations (Figure S3).

\section{The effect of ERß on OS}

We pooled the data from 11 studies $[1-5,10,15,18$ $20,23]$ with available overall survival data and observed that improved OS was associated with a positive status for pan-ER $\beta$ (HR=0.60, 95\%CI $0.45-0.80, P=0.0004$; heterogeneity: $\left.P=0.71, \mathrm{I}^{\wedge} 2=0 \%\right)$ and $\mathrm{ER} \beta-2(\mathrm{HR}=0.44$, 95\%CI 0.31-0.62, $P<0.0001$; heterogeneity: $P=0.90$, $\left.\mathrm{I}^{\wedge} 2=0 \%\right)$, but not $\mathrm{ER} \beta-1 \quad(\mathrm{HR}=0.55,95 \% \mathrm{CI} 0.20-1.50$, $P=0.24$; heterogeneity: $P<0.01, \mathrm{I}^{\wedge} 2=88 \%$; Figure 3 ). After excluding the study reported by Qui et al. [18], a positive ER $\beta-1$ status was shown to be associated with improved OS without significant heterogeneity $(\mathrm{HR}=0.38$, 
95\%CI $0.25-0.57$, heterogeneity: $P=1.00, \quad \mathrm{I}^{\wedge} 2=0 \%$ ). When the data from the two studies $[8,21]$ that used immunoblotting to assess pan-ER $\beta$ status were pooled, we observed an association between a positive pan-ER $\beta$ status and improved $\mathrm{OS}(\mathrm{HR}=0.62,95 \% \mathrm{CI} 0.46-0.84, P=0.002$; heterogeneity: $P=0.11, \mathrm{I}^{\wedge} 2=55 \%$; Figure $\left.\mathrm{S} 4\right)$. There were 3 studies [4, 20, 24] that assessed the mRNA levels of ER $\beta$ using PCR; we found no association between total ER $\beta$ mRNA levels and OS (Figure S5).

\section{$\mathrm{ER} \alpha$ as an effect modifier}

A total of 7 studies $[2,3,5,7,10,19,25]$ reported the HR of the IHC-determined ER $\beta$ status (pan-ER $\beta / E R \beta$ $1 / \mathrm{ER} \beta$-2) for DFS and OS in ER $\alpha$-positive or negative patient subgroups. In ER $\alpha(+)$ patients, $\mathrm{ER} \beta$ status was not associated with DFS (HR $=0.77,95 \%$ CI 0.46-1.27, $P=0.31$; heterogeneity: $\left.P=0.09, \mathrm{I}^{\wedge} 2=59 \%\right)$ or $\mathrm{OS}(\mathrm{HR}=0.64,95 \% \mathrm{CI}$ 0.37-1.11, $P=0.11$; heterogeneity: $P=0.09, \mathrm{I}^{\wedge} 2=54 \%$ ). In fact, Zhang [25] found that a positive $\operatorname{ER} \beta$ status was correlated with improved DFS in univariate, but not multivariate analysis. Vinayagam [4] reported that ER $\beta$ status was not correlated with DFS, but the associated HR was not available, and this study was therefore not included in the afore mentioned meta-analysis. In contrast, a positive ER $\beta$ status was significantly associated with increased DFS (HR=0.37, 95\%CI 0.14-0.93, $P=0.03$; heterogeneity: $\left.P<0.01, \mathrm{I}^{\wedge} 2=77 \%\right)$ and $\mathrm{OS}(\mathrm{HR}=0.44$, 95\%CI 0.30-0.65, $P<0.0001$; heterogeneity: $P=0.41$, $\mathrm{I}^{\wedge} 2=0 \%$ ) in $\operatorname{ER} \alpha(-)$ patients (Figures $4 \& 5$ ).

\section{Sensitivity analysis and publication bias}

A sensitivity analysis revealed that a positive $E R \beta$ (pan ER $\beta / E R \beta-1 / E R \beta-2$ ) status was significantly associated with improved DFS or OS in studies with a median follow-up time greater than 60 months [1-8, $10,15,17,19,20,22,23]$ (Table S1). ER $\beta-1$ was not associated with DFS or OS in studies with a sample size $\geq 200[2,5-8,17-19,25]$. The funnel plots for the studies

582 Potentially appropriate studies identified and screened for retrieval ( last literature search date: May 10, 2015 )

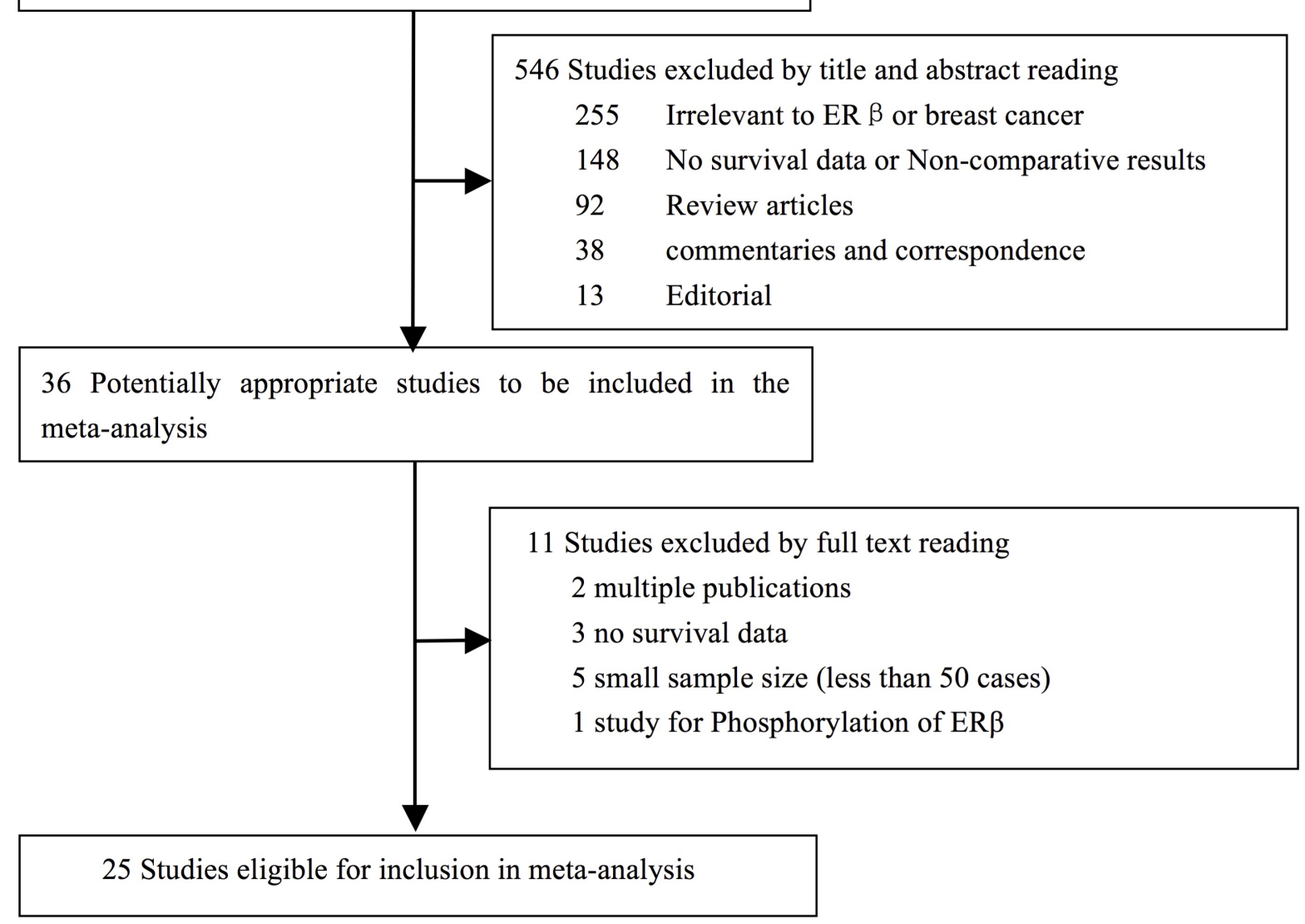

Figure 1: Flow diagram of studies identified, included, and excluded. 
for DFS were symmetric, indicating no publication bias (Figure S6). However, the distribution of the OS funnel plots was not symmetric. As shown in Figure S7, the studies focusing on specific $\operatorname{ER} \beta(\operatorname{ER} \beta 1 / \mathrm{ER} \beta 2)$ and pan$\mathrm{ER} \beta$ reactivity were mostly located on the left and right sides of the funnel plot, respectively, indicating possible publication biases.

\section{DISCUSSION}

ER $\beta$ was discovered nearly two decades ago, but its role as a prognostic or predictive factor in breast cancer remains elusive. Most studies examining ER $\beta$ as a biomarker have been retrospective, and these studies have used a variety of detection methods, leading to discrepant results. IHC is the most common method employed for ER $\beta$ assessment. In this meta-analysis study, we observed that a positive ER $\beta$ status, as assessed via IHC, was generally associated with improved DFS and OS. Multiple ER $\beta$ isoforms (ER $\beta-1, E R \beta-2 / c x)$ arise via alternative splicing of downstream coding exons or posttranslational proteolysis $[26,27]$. In this study, we noted that ER $\beta-2$ was associated with improved DFS and OS. In contrast, ER $\beta$-1 was associated with DFS, but not OS, which may be attributed to a study by Qui et al. [18], who provided the only report of an association between positive ER $\beta-1$ status and a poorer OS. After the exclusion of this study, the pooled $\mathrm{HR}(95 \% \mathrm{CI})$ of ER $\beta-1$ for OS changed significantly, from $0.55(95 \% \mathrm{CI}: 0.20-1.50)$ to $0.38(95 \%$ CI: $0.25-0.57)$. The heterogeneity of the data synthesis was also eliminated. After careful examination, we noted in consistent results within Qui et al.'s study. In their report, they indicated that ER $\beta$-positive patients exhibit a significantly worse overall survival prognosis compared with ER $\beta$-negative patients. However, when stratified by HER2 status, the survival curves of the ER $\beta$ positive and ER $\beta$-negative patients overlapped in both strata. The authors did not attempt to explain this result. We therefore suggest that the exclusion of this study from our meta-data analysis is appropriate.

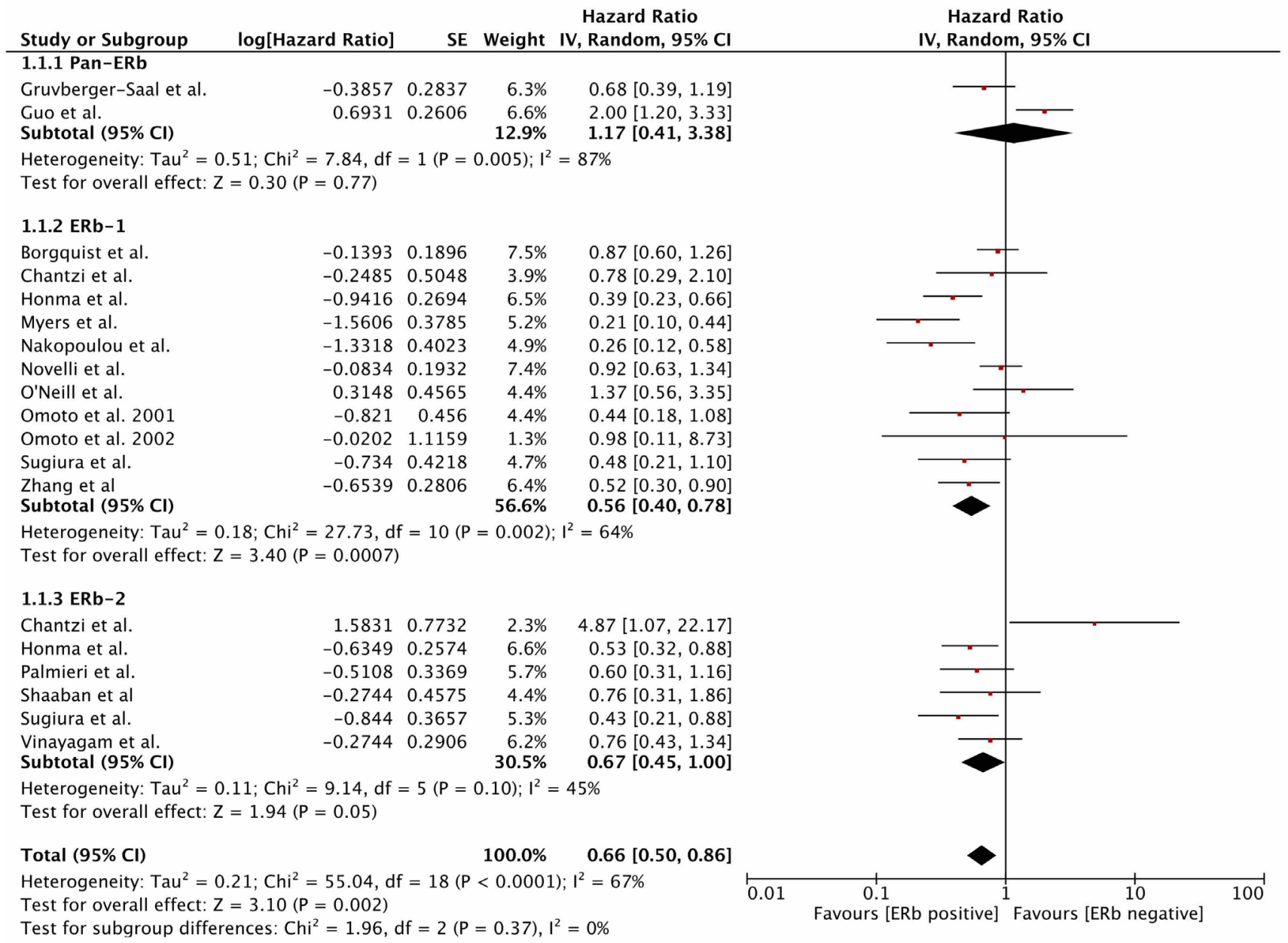

Figure 2: Prognostic role of IHC-determined ERß status for DFS. DFS, disease-free survival; IHC, immunohistochemistry; ER, estrogen receptor. 


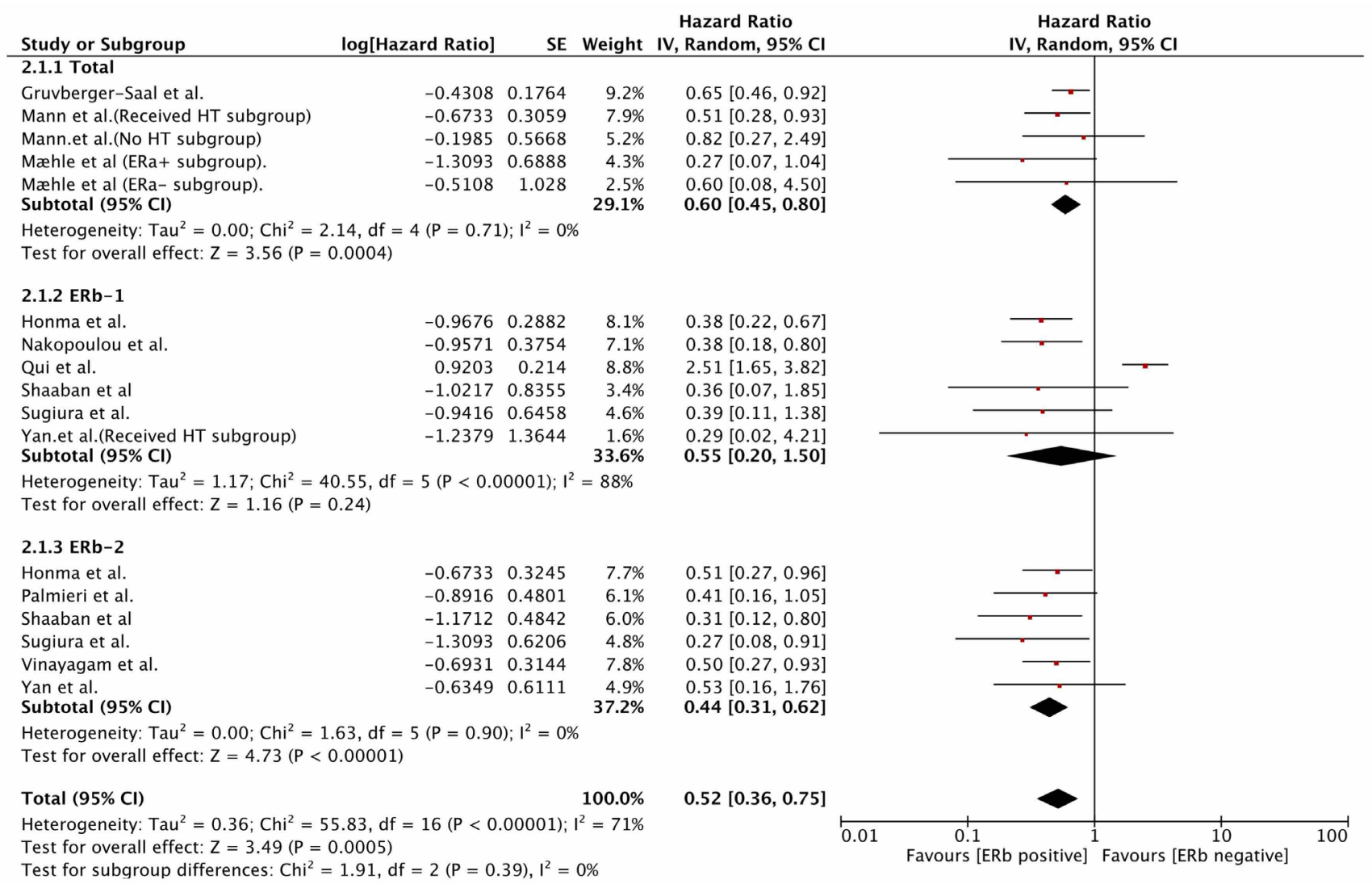

Figure 3: Prognostic role of IHC-determined ERß status for OS. OS, overall survival; IHC, immunohistochemistry; ER, estrogen receptor.

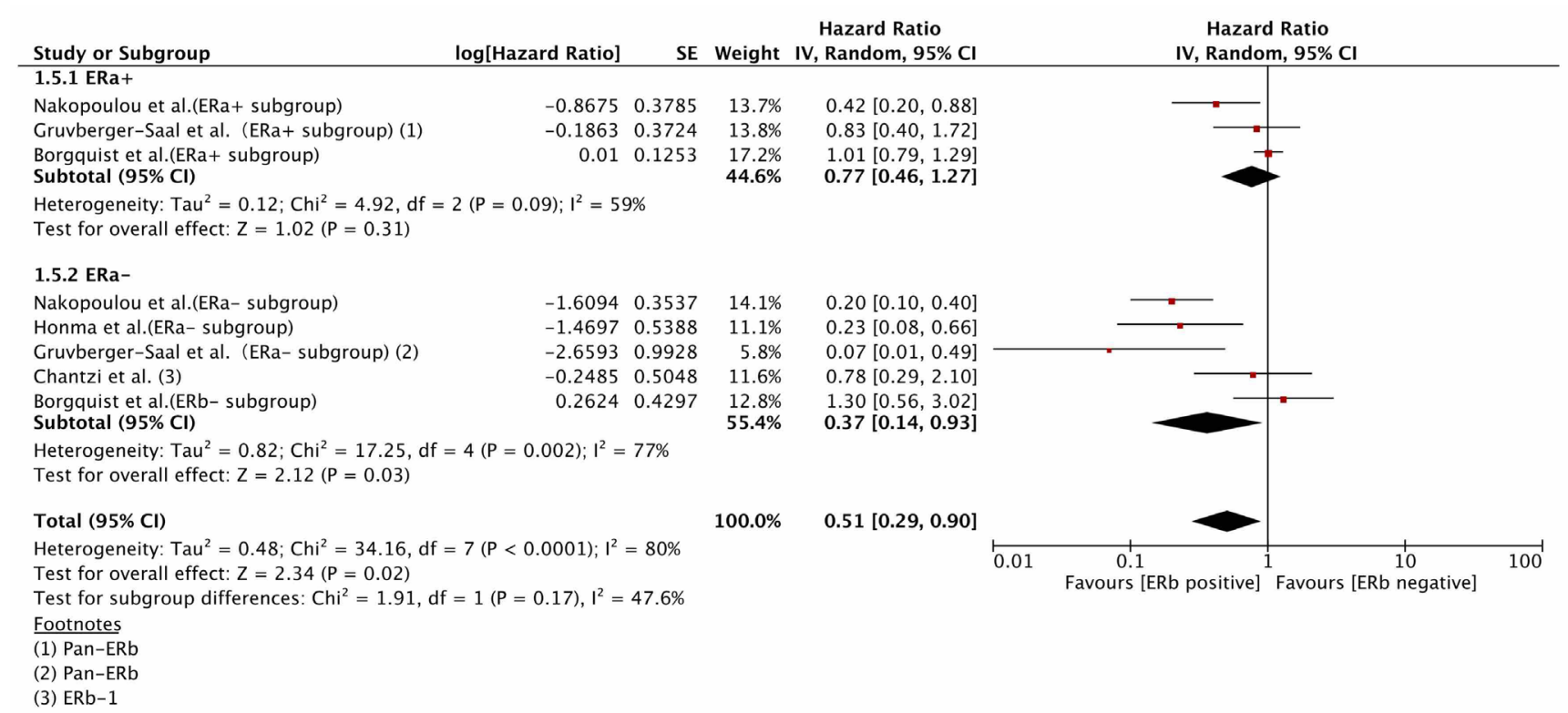

Figure 4: The prognostic role of IHC-determined ER $\beta$ status for DFS varied by ER $\alpha$ status. DFS, disease-free survival; IHC, immunohistochemistry; ER, estrogen receptor. 


\section{Assessment method and clinical outcomes}

Various methods had been used to assess ER $\beta$ status. Two studies employed immunoblotting as the detection method and revealed that a positive ER $\beta$ status was associated with improved DFS, similar to studies employing IHC $[8,21]$. However, a study by Wimberly et al. [22] showed no association between ER $\beta$ status and DFS when TMA was used to assess ER $\beta$ status in four largecohort populations. We speculate that TMA may not be an accurate method for ER $\beta$ assessment. Its major limitation is that the small cores employed to construct a TMA may not accurately and comprehensively represent the whole tissue specimen. Eckel-Passow et al. [28]reported that the number of TMA cores necessary to adequately represent the whole tissue specimen is biomarker-specific. They showed that 2-3 cores appeared to be adequate for assessing the status of B7-H3, Ki-67, CAIX, and IMP3 expression in renal cancer patients, whereas as many as 10 cores were insufficient for assessing B7-H1. Thus, the association between $\mathrm{B} 7-\mathrm{H} 1$ determined in whole tissue sections and renal cancer-specific death is not easily revealed through TMA assessment.

Several studies found no consistent association between the mRNA and protein levels of $\operatorname{ER} \beta[14,29,30]$. Furthermore, an inverse association between ER $\beta$ mRNA levels and improved survival has been reported. Speirs et al. [31]noted that ER $\beta$ mRNA levels were increased in tamoxifen-resistant breast cancer patients. Similarly, Kim et al. [9] reported that a higher ER $\beta$ mRNA level is associated with poorer DFS in patients treated using endocrine therapy. We believe that the assessment of ER $\beta$ status based on mRNA levels may be inaccurate because samples from breast tissue might contain cells from surrounding cancerous tissue. Furthermore, post- transcriptional regulation may also compromise the prognostic value of ER $\beta$ mRNA [32]. In our meta-analysis, we found no association between ER $\beta$ mRNA levels and survival (DFS or OS). Hence, ER $\beta$ mRNA status does not appear to be promising for clinical use.

\section{Prognostic role of ER $\beta$ varied by ER $\alpha$ status}

As noted above, the prognostic value of ER $\beta$ varies depending on a patient's ER $\alpha$ status. The mechanism underlying this effect may be the molecular interplay between ER $\alpha$ and ER $\beta$. Charn et al. [33] investigated the effects of ligand-occupied and unoccupied ER $\alpha$ and ER $\beta$ on chromatin binding. They showed that although ER $\alpha$ and ER $\beta$ restrict each other's binding site occupancy, ER $\alpha$ is dominant. The binding sites of ER $\alpha$ and $E R \beta$ overlap substantially when the are present alone. However, when both ER $\alpha$ and ER $\beta$ are present, only a few binding sites are shared. When both receptors are present, ER $\alpha$ displaces ER $\beta$ and shifts ligand binding to sites that are less enriched in the estrogen response element. This finding supports our observation that in ER $\alpha+$ patients, the prognostic role of ER $\beta$ was less significant than in ER $\alpha$ - patients. Because endocrine therapy is administered to $\mathrm{ER} \alpha+$, but not ER $\alpha$-, patients, we suggest that endocrine therapy may play a role as an effect modifier. Unfortunately, there are insufficient data to perform a meta-analysis addressing this issue. Novelliet al.[6]reported that in patients who receive endocrine therapy, a positive ER $\beta$ status is associated with increased DFS. Similar results have been reported by other investigators [1, 8]. However, Yan et al. [23] found that a positive ER $\beta$ status was associated with improved OS in univariate, but not multivariate, analyses. O'Neil et al. [12]noted a trend (though not statistically significant) toward poorer DFS in patients with a positive ER $\beta$ status.

\begin{tabular}{|c|c|c|c|c|c|c|c|c|}
\hline Study or Subgroup & log[Hazard Ratio] & SE & Weight & $\begin{array}{l}\text { Hazard Ratio } \\
\text { IV, Random, } 95 \% \mathrm{Cl}\end{array}$ & & $\begin{array}{r}\text { Hazard } \\
\text { IV, Randor }\end{array}$ & $\begin{array}{l}\text { d Ratio } \\
\mathrm{m}, 95 \% \mathrm{Cl}\end{array}$ & \\
\hline \multicolumn{9}{|l|}{ 2.4.1 ERa+ } \\
\hline Gruvberger-Saal et al. (ERa+ subgroup) & 0 & 0.182 & $20.2 \%$ & $1.00[0.70,1.43]$ & & & & \\
\hline Gruvberger-Saal et al. (ERa- subgroup) & 0 & 0 & & Not estimable & & & & \\
\hline Mæhle et al (ERa+ subgroup). & -1.3093 & 0.6888 & $6.0 \%$ & $0.27[0.07,1.04]$ & & & & \\
\hline Nakopoulou et al.(ERa+ subgroup) & -0.3285 & 0.398 & $12.1 \%$ & $0.72[0.33,1.57]$ & & & 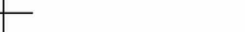 & \\
\hline $\begin{array}{l}\text { Shaaban et al.(ERa+ subgroup) } \\
\text { Subtotal }(95 \% \mathrm{Cl})\end{array}$ & -0.9163 & 0.4366 & $\begin{array}{l}11.0 \% \\
49.3 \%\end{array}$ & $\begin{array}{l}0.40[0.17,0.94] \\
0.64[0.37,1.11]\end{array}$ & & & & \\
\hline \multicolumn{9}{|c|}{$\begin{array}{l}\text { Heterogeneity: } \mathrm{Tau}^{2}=0.17 ; \mathrm{Chi}^{2}=6.58, \mathrm{df}=3(\mathrm{P}=0.09) ; \mathrm{I}^{2}=54 \% \\
\text { Test for overall effect: } \mathrm{Z}=1.59(\mathrm{P}=0.11)\end{array}$} \\
\hline \multicolumn{9}{|l|}{ 2.4.2 ERa- } \\
\hline Gruvberger-Saal et al. (ERa- subgroup) & -1.1394 & 0.3866 & $12.5 \%$ & $0.32[0.15,0.68]$ & & & & \\
\hline Honma et al.(ERa- subgroup) & -1.2946 & 0.5568 & $8.2 \%$ & $0.27[0.09,0.82]$ & & & & \\
\hline Mæhle et al (ERa- subgroup). & -0.5108 & 1.028 & $3.1 \%$ & $0.60[0.08,4.50]$ & & & & \\
\hline Nakopoulou et al.(ERa- subgroup) & -0.9676 & 0.3812 & $12.6 \%$ & $0.38[0.18,0.80]$ & & & & \\
\hline $\begin{array}{l}\text { Shaaban et al.(ERa- subgroup) } \\
\text { Subtotal }(95 \% \mathrm{Cl})\end{array}$ & -0.3147 & 0.3331 & $\begin{array}{l}14.3 \% \\
\mathbf{5 0 . 7 \%}\end{array}$ & $\begin{array}{l}0.73[0.38,1.40] \\
0.44[0.30,0.65]\end{array}$ & & & & \\
\hline \multicolumn{9}{|c|}{$\begin{array}{l}\text { Heterogeneity: } \operatorname{Tau}^{2}=0.00 ; \mathrm{Chi}^{2}=3.95, \mathrm{df}=4(\mathrm{P}=0.41) ; \mathrm{I}^{2}=0 \% \\
\text { Test for overall effect: } Z=4.21(\mathrm{P}<0.0001)\end{array}$} \\
\hline Total $(95 \% \mathrm{CI})$ & & & $100.0 \%$ & $0.52[0.36,0.76]$ & & & & \\
\hline \multicolumn{5}{|c|}{$\begin{array}{l}\text { Heterogeneity: } \mathrm{Tau}^{2}=0.16 ; \mathrm{Chi}^{2}=16.39, \mathrm{df}=8(\mathrm{P}=0.04) ; \mathrm{I}^{2}=51 \% \\
\text { Test for overall effect: } \mathrm{Z}=3.34(\mathrm{P}=0.0008) \\
\text { Test for subgroup differences: } \mathrm{Chi}^{2}=1.11, \mathrm{df}=1(\mathrm{P}=0.29), \mathrm{I}^{2}=10.2 \%\end{array}$} & 0.01 & $\begin{array}{c}0.1 \\
\text { Favours [ERb positive] }\end{array}$ & Favours [ERb negative] & 100 \\
\hline
\end{tabular}

Figure 5: The prognostic role of IHC-determined ERß status for OS varied by ERa status. OS, overall survival; IHC, immunohistochemistry; ER, estrogen receptor. 


\begin{tabular}{|c|c|c|c|c|c|c|c|c|}
\hline \multicolumn{9}{|c|}{ Table 1b: Features of included studies } \\
\hline \multirow{2}{*}{ References } & \multirow{2}{*}{ Year } & \multirow{2}{*}{ Patients (n) } & \multirow{2}{*}{ Antibody } & \multicolumn{2}{|c|}{ ERa status } & \multicolumn{3}{|c|}{ Tumor Burden } \\
\hline & & & & ER $\alpha+$ & $\mathrm{ER} \alpha-$ & T1\% & \multirow{2}{*}{\begin{tabular}{|l|}
$\mathrm{N} 0 \%$ \\
$63.1 \%$ \\
\end{tabular}} & \multirow{2}{*}{\begin{tabular}{|l|} 
G3 $\%$ \\
NA \\
\end{tabular}} \\
\hline \multirow{3}{*}{$\begin{array}{l}\text { Borgquist et } \\
\text { al.[11] }\end{array}$} & \multirow{3}{*}{2008} & 512 & \multirow{3}{*}{$\begin{array}{lrr}\text { ER } \beta 1: & \text { anti-mouse } & \text { ER } \beta 1 \\
\text { monoclonal antibody } & \text { (EMR02; } \\
\text { Novocastra) } & & \\
\end{array}$} & & & $63.1 \%$ & & \\
\hline & & \begin{tabular}{|l|l}
$114 ~ \#$ \\
\end{tabular} & & \begin{tabular}{|l|}
40 \\
95 \\
11
\end{tabular} & & NA & NA & NA \\
\hline & & 139 \#\# & & 114 & 25 & NA & NA & NA \\
\hline $\begin{array}{l}\text { Chantzi et } \\
\text { al.[20] }\end{array}$ & 2013 & 95 & $\begin{array}{l}\text { ER } \beta 1 \text { :anti-mouse ER } \beta 1 \text { monoclonal } \\
\text { antibody (Clone PPG5/10; Serotec) } \\
\text { ER } \beta 2 / c x \text { anti-human ER } \beta 2 \\
\text { monoclonal antibody (Clone \# 57/3; } \\
\text { Serotec) } \\
\end{array}$ & 0 & 95 & $44.2 \%$ & $56.8 \%$ & $47.4 \%$ \\
\hline 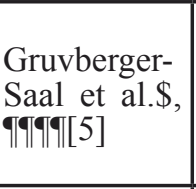 & 2007 & 425 & $\begin{array}{lr}\text { Pan-ER } \beta \text { :anti-mouse } & \text { ER } \beta \\
\text { monoclonal } & \text { antibody } \\
\text { (Clone 14C8; } & \text { GeneTex) } \\
\text { ER } \beta 1: \text { anti-mouse ER } \beta 1 \text { monoclonal } \\
\text { antibody (Clone PPG5/10; Serotec) }\end{array}$ & 248 & 105 & $26.6 \%$ & $33.4 \%$ & NA \\
\hline $\begin{array}{l}\text { Guo et al. [2, } \\
21] \pi \uparrow \uparrow \uparrow \uparrow\end{array}$ & 2014 & 490 & $\begin{array}{l}\text { Pan-ERß: Unclear (Fuzhou Maixin } \\
\text { Biotechnology Development) }\end{array}$ & NA & NA & $32.8 \%$ & $51.2 \%$ & $26.1 \%$ \\
\hline $\begin{array}{l}\text { Honma et al. } \\
\S[2]\end{array}$ & 2008 & 442 & $\begin{array}{l}\text { Pan-ERß:anti-rabbit polyclonal } \\
\text { antibody (MYEB, M.Y) } \\
\text { ER } \beta 1: \text { anti-mouse ER } \beta 1 \text { monoclonal } \\
\text { antibody (Clone PPG5/10; DAKO) } \\
\text { ER } \beta 2 / \text { cx:anti-mouser ER } \beta 2 \\
\text { monoclonal antibody (Clone \# 57/3; } \\
\text { Serotec) } \\
\end{array}$ & 364 & 78 & $39.4 \%$ & $54.8 \%$ & NA \\
\hline & & 305 & nti-mouse & 272 & 33 & $23.9 \%$ & $0.0 \%$ & $43.7 \%$ \\
\hline $\begin{array}{l}\text { Hopp } \\
\text { al [12] }\end{array}$ & 2004 & \begin{tabular}{|l|l}
$186 \#$ \\
\end{tabular} & monoclonal antibody (Clone 14C8; & 176 & 10 & $26.9 \%$ & NA & $40.7 \%$ \\
\hline & & 119 \#\# & & 96 & 23 & $19.5 \%$ & NA & $48.3 \%$ \\
\hline $\begin{array}{ll}\text { Kim } & \text { et } \\
\text { al.[13] } & \\
\end{array}$ & 2012 & 139 & NA & 139 & 0 & $61.4 \%$ & $42.4 \%$ & $20.7 \%$ \\
\hline \begin{tabular}{ll|} 
Mahle & et \\
al. $\$[14]$
\end{tabular} & 2009 & 145 & $\begin{array}{l}\text { Pan-ER } \beta \text { :anti-mouse } \\
\text { monoclonal antibody (Clone 14C8; } \\
\text { GeneTex) } \\
\end{array}$ & 97 & 48 & $37.0 \%$ & $51.7 \%$ & $24.3 \%$ \\
\hline Mann & 2001 & 47 \#\# & Pan-ERß:anti-rabbit polyclonal & 30 & 17 & NA & NA & NA \\
\hline al.[1] & 2001 & 118 \# & antibc & 75 & 43 & NA & $100.0 \%$ & NA \\
\hline $\begin{array}{l}\text { Markey et } \\
\text { al.[28] }\end{array}$ & 2009 & 121 & NA & 82 & 36 & $32.2 \%$ & $45.5 \%$ & $43.0 \%$ \\
\hline $\begin{array}{ll}\text { Myers } & \text { et } \\
\text { al.[15] }\end{array}$ & 2004 & 150 & $\begin{array}{l}\text { ER } \beta 1 \text { :anti-mouse ER } \beta 1 \text { monoclonal } \\
\text { antibody (Clone PPG5/10; Serotec) }\end{array}$ & 123 & 27 & NR & $37.3 \%$ & $49.3 \%$ \\
\hline $\begin{array}{l}\text { Nakopoulou } \\
\text { et al.[3] }\end{array}$ & 2004 & 181 & $\begin{array}{l}\text { ER } \beta 1 \text { :anti-mouse ER } \beta 1 \text { monoclonal } \\
\text { antibody (Clone PPG5/10; Serotec) }\end{array}$ & 117 & 61 & $27.1 \%$ & $38.1 \%$ & $29.3 \%$ \\
\hline $\begin{array}{l}\text { Novelli et } \\
\text { al.[6] }\end{array}$ & 2008 & 936 & 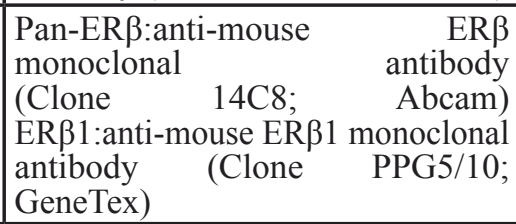 & 658 & 278 & $61.9 \%$ & $57.6 \%$ & $31.2 \%$ \\
\hline $\begin{array}{ll}\text { Omoto } & \text { et } \\
\text { al.[18] } & \end{array}$ & 2002 & 57 & 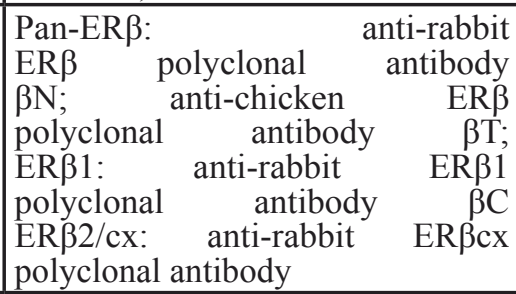 & 39 & 18 & $21.1 \%$ & $62.5 \%$ & $14.0 \%$ \\
\hline $\begin{array}{ll}\text { Omoto } \\
\text { al.[17] }\end{array}$ & 2001 & 88 & $\begin{array}{l}\text { ER } \beta 1: \text { anti-rabbit ER } \beta 1 \text { polyclonal } \\
\text { antibody } \beta C\end{array}$ & 62 & 26 & $22.7 \%$ & $52.3 \%$ & $4.5 \%$ \\
\hline
\end{tabular}




\begin{tabular}{|c|c|c|c|c|c|c|c|c|}
\hline \multirow{2}{*}{$\begin{array}{l}\text { O'Neill et } \\
\text { al.q[16] }\end{array}$} & \multirow{2}{*}{2004} & \multirow[t]{2}{*}{167} & $\begin{array}{l}\text { ER } \beta 1 \text { :anti-mouse ER } \beta 1 \text { monoclonal } \\
\text { antibody (Clone PPG5/10; Serotec) }\end{array}$ & 83 & 44 & \multirow{2}{*}{$40.6 \%$} & \multirow{2}{*}{$53.3 \%$} & \multirow{2}{*}{$45.5 \%$} \\
\hline & & & \begin{tabular}{|l} 
NA \\
\end{tabular} & 79 & 42 & & & \\
\hline $\begin{array}{l}\text { Palmieri et } \\
\text { al.[19] }\end{array}$ & 2004 & 82 & $\begin{array}{|lr|}\text { Pan-ER } \beta: \text { a } & \text { purified } \\
\text { polyclonal } & \text { antibody } \\
\text { ER } \beta 2 / \text { cx: anti-ERßcx } & \text { sheep } \\
\text { polyclonal antibody } & \\
\end{array}$ & 46 & 33 & $25.7 \%$ & $53.2 \%$ & $40.7 \%$ \\
\hline $\begin{array}{ll}\text { Qui } & \text { et } \\
\text { al.[22] } & \end{array}$ & 2009 & 308 & $\begin{array}{l}\text { ER } \beta 1 \text { :anti-rabbit ER } \beta \text { polyclonal } \\
\text { antibody(Ab-1, Oncogene research } \\
\text { product) }\end{array}$ & 198 & 110 & $\begin{array}{l}42.2 \% \\
\& \&\end{array}$ & $37.8 \%$ & $39.6 \%$ \\
\hline $\begin{array}{l}\text { Shaaban } \\
\text { al.[23] }\end{array}$ & 2008 & 880 & $\begin{array}{l}\text { ER } \beta 1 \text { :anti-mouse ER } \beta 1 \text { monoclonal } \\
\text { antibody (Clone PPG5/10; Serotec) } \\
\text { ER } \beta 2 / \text { cx: anti-human ER } \beta 2 \\
\text { monoclonal antibody (Clone \# 57/3; } \\
\text { Serotec) }\end{array}$ & 451 & 219 & NA & NA & $45.8 \%$ \\
\hline \multirow[t]{2}{*}{$\begin{array}{l}\text { Sugiura et } \\
\text { al.[24] }\end{array}$} & \multirow[t]{2}{*}{2007} & \multirow[t]{2}{*}{150} & \begin{tabular}{|lrr} 
ERß1: & anti-rabbit & ER $\beta 1$ \\
polyclonal & & antibody \\
ER $\beta 2 /$ cx: & anti-rabbit & ER $\beta 2 / c x$ \\
polyclonal antibody & \\
\end{tabular} & 117 & 33 & $27.3 \%$ & $60.4 \%$ & $25.2 \%$ \\
\hline & & & NA & 117 & 33 & $27.3 \%$ & $60.4 \%$ & $25.2 \%$ \\
\hline \multirow[t]{2}{*}{$\begin{array}{l}\text { Vinayagam } \\
\text { et al. } \uparrow, \S[4]\end{array}$} & \multirow[t]{2}{*}{2007} & 141 & $\begin{array}{l}\text { ER } \beta 2 / c x: \text { anti-human } \quad \text { ER } \beta 2 \\
\text { monoclonal antibody (Clone \# 57/3; } \\
\text { Serotec) }\end{array}$ & 98 & 43 & $44.7 \%$ & $47.5 \%$ & $43.3 \%$ \\
\hline & & 100 & NA & 70 & 30 & $44.0 \%$ & $49.0 \%$ & $42.0 \%$ \\
\hline $\begin{array}{|ll|}\begin{array}{l}\text { Wen } \\
\text { al.[25] }\end{array} & \text { et } \\
\end{array}$ & 2002 & 116 & $\begin{array}{l}\text { Pan-ER } \beta \text { : anti-goat ER } \beta \text { polyclonal } \\
\text { antibody(Santa Cruz) }\end{array}$ & 73 & 43 & $12.9 \%$ & $37.1 \%$ & $38.8 \%$ \\
\hline \multirow{4}{*}{$\begin{array}{l}\text { Wimberly et } \\
\text { al. [26] }\end{array}$} & \multirow{4}{*}{2014} & Yale-1:649 & \multirow{4}{*}{ 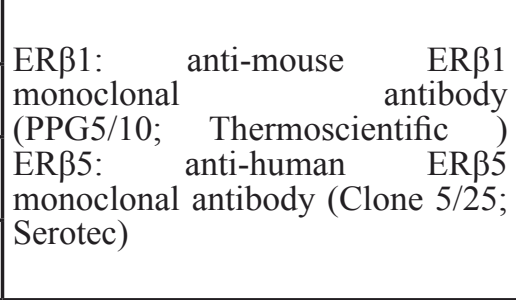 } & 246 & 208 & $28.0 \%$ & $42.6 \%$ & \multirow{4}{*}{ NA } \\
\hline & & Yale-2:398 & & 158 & 102 & $54.8 \%$ & $51.4 \%$ & \\
\hline & & Toronto: 976 & & 288 & 118 & $65.3 \%$ & $100.0 \%$ & \\
\hline & & $\begin{array}{l}\text { NCI-PBCS: } \\
1375\end{array}$ & & 656 & 271 & $52.7 \%$ & $59.0 \%$ & \\
\hline $\begin{array}{lr}\text { Yan } & \text { et } \\
\text { al. } \\
\text { aाף }\end{array}$ & 2011 & 147 & 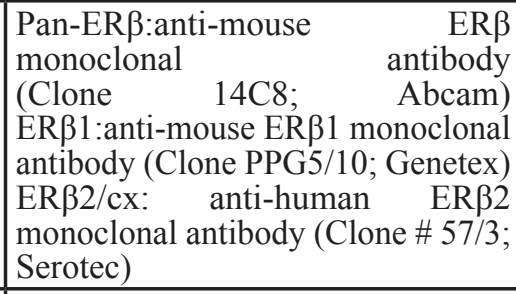 & 62 & 48 & $55.3 \%$ & $69.9 \%$ & $64.4 \%$ \\
\hline $\begin{array}{ll}\text { Zhang } & \text { et } \\
\text { al.[29] } & \end{array}$ & 2014 & 279 & $\begin{array}{l}\text { ER } \beta 1 \text { :anti-mouse ER } \beta 1 \text { monoclonal } \\
\text { antibody (Clone PPG5/10; Serotec) } \\
\text { ER } \beta 2 / \text { cx: anti-human ER } \beta 2 \\
\text { monoclonal antibody (Clone \# 57/3; } \\
\text { Serotec) }\end{array}$ & 131 & 21 & $70.5 \%$ & $41.6 \%$ & $24.8 \%$ \\
\hline \multicolumn{9}{|c|}{ 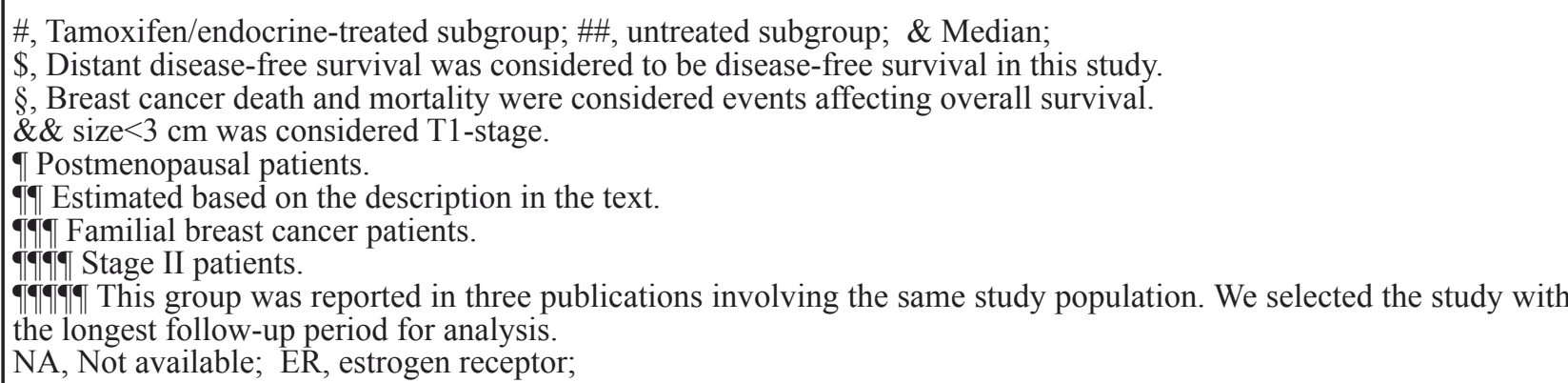 } \\
\hline
\end{tabular}


Hence, the predictive role of $\operatorname{ER} \beta$ for the endocrine therapy response is unclear, due to the conflicting results provided by different studies $[1,6,7,12,23]$.

No association between ER $\beta$ status and DFS/OS was observed in patients who did not receive endocrine therapy $[1,7,23]$. We believe that the sample sizes of these studies are too small to detect an association. Our group has initiated a multicenter randomized doubleblind prospective clinical trial comparing the efficacy of tamoxifen as an adjuvant endocrine therapy in early-stage $\mathrm{ER} \alpha / \mathrm{PR}-/ \mathrm{ER} \beta+$ breast cancer patients (ClinicalTrials.gov Identifier:NCT02062489). Sun et al. has initiated a similar multicenter study, in which early stage, triple-negative breast cancer patients are randomized into a toremifene/ anastrozole group or an observation group (Clinical Trials. gov Identifier: NCT02089854)

\section{DFS and OS as clinical endpoints}

We observed heterogeneity in the synthesis of the HR of pan-ER $\beta$ or ER $\beta-1$ status for DFS. However, there was no heterogeneity in the synthesis of the HR of pan$E R \beta, E R \beta-1$ (with the exception of Qui's study) or ER $\beta$ 2 status for OS. We suggest that this discrepancy may be due to the definition of DFS/OS. OS is a universally accepted measure of the clinical benefit of a treatment and can be precisely measured. As a result, there might be less heterogeneity for OS. In contrast, the definition of DFS varies between studies. For example, in the NSABP B-06 study [34], DFS was defined as the first recurrence of disease at a local, regional, or distant site, and the diagnosis of a second cancer and death without evidence of cancer were considered DFS events. In contrast, the guidelines from the DATECAN initiative (Definition for the Assessment of Time-to-event Endpoints in CANcer trials) [35] recommend that DFS should include death of from breast cancer as an event. Most of our included studies did not specify the definition of DFS, which may have resulted in heterogeneity in the synthesis of HR of DFS.

\section{Publication bias}

All of the included studies were retrospective and may be subject to publication bias. Insignificant HRs, especially following multivariate analysis, are less likely to be reported in retrospective studies. In the present study, we obtained asymmetric funnel plots for the synthesis of the HRs for OS. Studies reported significant HRs of ER $\beta$ 1 and ER $\beta$ - 2 for OS tend to fall on the left side of the reference line, indicating that insignificant HRs are less likely to be reported. Several studies $[4,6,23,36]$ reported finding no association between ER $\beta$ status and OS, but without an available HR and/or 95\%CI. Hence, we must be cautious about the prognostic role of ER $\beta$ for overall survival.

\section{Limitations}

Several additional limitations should be addressed. First, IHC was commonly used for detecting ER $\beta$ status in most studies, but different hospital used varied commercial antibodies and didn't have uniform criteria. Reported data show that many commercially available IHC stains for ER $\beta$ have cross-reactivity with ER $\alpha$ [37]. Percentage of immunoreactive cells and allred scores were used to assess $\mathrm{ER} \beta$ status, while the cut-off values varied from $1 \%$ to $25 \%$ (Percentage of immunoreactive cells) [6, 14, 17], and from 2-4 (Allred score) $[2,11,15]$ across different studies. Different cut-off values used by different studies may cause limitation to our analysis. Second, some of the HRs were not available from the full-text of the included study, and were extrapolated from survival curves. Although, this method has been demonstrated to be feasible [38, 39], we still consider this as a limitation. Additionally, HRs for synthesis in our analysis were derived from univariate and/or multivariate analysis (Table S2). This is also a major limitation, as the most standard approach should be collecting HRs derived from prospective controlled trials, with multivariate analysis adjustment.

\section{CONCLUSION}

In this meta-analysis, we showed that $\operatorname{ER} \beta$ status, determined via IHC, is generally associated with DFS/OS in breast cancer patients. Assessment of ER $\beta$ mRNA levels is not recommended. As a prognostic factor, ER $\beta$ may be more important in $\mathrm{ER} \alpha(+)$ patients than $\mathrm{ER} \alpha(-)$ patients. Based on these findings, we recommend the initiation of a prospective study to confirm the prognostic value of ER $\beta$ in breast cancer patients.

\section{MATERIALS AND METHODS}

This study was waived the full IRB review of Sun Yat-sen Memorial Hospital, based on the institutional policy. This study was also performed according to the recommendations of the Cochrane Collaboration and the Quality of Reporting of Meta-analysis guidelines (MOOSE) and reported according to the PRISMA statements $[40,41]$.

\section{Study selection}

We searched Medline, Embase, and the Web of Science for potentially relevant studies. The following keywords were searched in the "Title" or "Abstract": "Estrogen receptor," "Beta," and "Breast cancer," without restrictions on the region and publication type. English language was requied for publication. We manually 
searched the retrieved articles to identify relevant studies. When multiple publications reported on the same study population, the report that was most complete or that had the longest follow-up period was used. The last date of the search was May 10 $0^{\text {th }}, 2015$.

\section{Inclusion and exclusion criteria}

Studies were eligible if they met the following criteria: (1) the main exposure of interest was early-stage breast cancer stratified by ER $\beta$ status (negative/positive or low/high expression); (2) the outcome of interest was disease-free survival or overall survival; (3) hazard ratios (HRs) with corresponding 95\% confidence intervals (CIs) or survival curves for ER $\beta$ were reported; and (4) over 50 patients were enrolled in the study, which did not present redundant data.

\section{Data extraction}

Two reviewers (C.G. \& W.T.) independently extracted the data from the included studies. Any disagreement was resolved by the third author (E.S.). The following data were collected: first author, year of publication, clinicopathological features of the study population, methods of ER $\beta$ assessment, number of included patients, and the reported outcomes. The outcomes assessed included disease-free survival (DFS) and overall survival (OS) in patients with different ER $\beta$ statuses. We assessed the quality of the included studies using the Newcastle-Ottawa quality assessment tool [42]. We allocated a score of 0-9 to each included study, and those with a score $\geq 6$ were considered to be of high quality.

\section{Statistical analysis}

The hazard ratio (HR) was used as a summary statistic for survival analysis, as described by Parmar and colleagues [43]. An HR of less than 1 indicated a survival benefit favoring ER $\beta+$ patients. We used a random-effects model for this meta-analysis. The data were pooled and weighted using generic inverse variance. Heterogeneity between studies was assessed with the $\chi 2$ and 12 statistics. When higher values of the $\chi 2$ and I2 statistics ( $>50 \%$ ) indicated heterogeneity between studies, we applied sensitivity and subgroup analyses to further evaluate the heterogeneity. We performed a sensitivity analysis when the outcome of interest was reported in more than 3 studies. We used funnel plot analyses to analysis to determine publication bias. A two-tailed $p$ value of less than 0.05 was considered statistically significant. Statistical analyses were performed with Review Manager Version 5.3

\section{ACKNOWLEDGMENTS}

We appreciate Yilong Education, Inc, for advices of statistical analysis.

\section{CONFLICTS OF INTEREST}

\author{
None to disclose.
}

\section{GRANT SUPPORT}

This work was funded by grants from Natural Science Foundation of China (81272893, 81472466 ,81402201,81172514,81372817 81230060, 81490750, 81442009); Program for New Century Excellent Talents in University (NCET-12-0565); National Science Foundation of Guangdong Province Grants (2014A03036003, 2014A030310070, S2012030006287, 4202037, 2011A080300002), Guangzhou Science Technology and Innovation Commission (201508020008, 201508020249), Guangdong Science and Technology Department (2015B050501004), Elite Young Scholars Program of Sun Yat-sen Memorial Hospital (Y201401). Grant KLB09001 from the Key Laboratory of Malignant Tumor Gene Regulation and Target Therapy of Guangdong Higher Education Institutes, Sun-Yat-Sen University, Grant [2013]163 from Key Laboratory of Malignant Tumor Molecular Mechanism and Translational Medicine of Guangzhou Bureau of Science and Information Technology.

\section{REFERENCES}

1. Mann S, Laucirica R, Carlson N, Younes PS, Ali N, Younes A, Li Y and Younes M. Estrogen receptor beta expression in invasive breast cancer. Human pathology. 2001; 32:113118.

2. Honma N, Horii R, Iwase T, Saji S, Younes M, Takubo K, Matsuura M, Ito Y, Akiyama F and Sakamoto G. Clinical importance of estrogen receptor-beta evaluation in breast cancer patients treated with adjuvant tamoxifen therapy. J Clin Oncol. 2008; 26:3727-3734.

3. Nakopoulou L. The favourable prognostic value of oestrogen receptor immunohistochemical expression in breast cancer. Journal of clinical pathology. 2004; 57:523528.

4. Vinayagam R, Sibson DR, Holcombe C, Aachi V and Davies MP. Association of oestrogen receptor beta 2 (ER beta $2 /$ ER beta $\mathrm{cx}$ ) with outcome of adjuvant endocrine treatment for primary breast cancer--a retrospective study. BMC cancer. 2007; 7:131.

5. Gruvberger-Saal SK, Bendahl PO, Saal LH, Laakso M, Hegardt C, Eden P, Peterson C, Malmstrom P, Isola J, Borg A and Ferno M. Estrogen receptor beta expression is associated with tamoxifen response in ERalpha-negative 
breast carcinoma. Clin Cancer Res. 2007; 13:1987-1994.

6. Novelli F, Milella M, Melucci E, Di Benedetto A, Sperduti I, Perrone-Donnorso R, Perracchio L, Venturo I, Nistico C, Fabi A, Buglioni S, Natali PG and Mottolese M. A divergent role for estrogen receptor-beta in node-positive and node-negative breast cancer classified according to molecular subtypes: an observational prospective study. Breast Cancer Res. 2008; 10:R74.

7. Borgquist S, Holm C, Stendahl M, Anagnostaki L, Landberg $\mathrm{G}$ and Jirstrom K. Oestrogen receptors alpha and beta show different associations to clinicopathological parameters and their co-expression might predict a better response to endocrine treatment in breast cancer. Journal of clinical pathology. 2008; 61:197-203.

8. Hopp TA, Weiss HL, Parra IS, Cui Y, Osborne CK and Fuqua SA. Low levels of estrogen receptor beta protein predict resistance to tamoxifen therapy in breast cancer. Clin Cancer Res. 2004; 10:7490-7499.

9. Kim TJ, Lee A, Choi YJ, Song BJ, Yim HW and Kang CS. Prognostic Significance of High Expression of ER-beta in Surgically Treated ER-Positive Breast Cancer Following Endocrine Therapy. Journal of breast cancer. 2012; 15:7986.

10. Maehle BO, Collett K, Tretli S, Akslen LA and Grotmol T. Estrogen receptor beta--an independent prognostic marker in estrogen receptor alpha and progesterone receptorpositive breast cancer? APMIS. 2009; 117:644-650.

11. Myers E, Fleming FJ, Crotty TB, Kelly G, McDermott EW, O'Higgins N J, Hill AD and Young LS. Inverse relationship between ER-beta and SRC-1 predicts outcome in endocrineresistant breast cancer. British journal of cancer. 2004; 91:1687-1693.

12. O'Neill PA, Davies MP, Shaaban AM, Innes H, Torevell A, Sibson DR and Foster CS. Wild-type oestrogen receptor beta (ERbeta1) mRNA and protein expression in Tamoxifen-treated post-menopausal breast cancers. British journal of cancer. 2004; 91:1694-1702.

13. Omoto $\mathrm{Y}$, Inoue $\mathrm{S}$, Ogawa $\mathrm{S}$, Toyama $\mathrm{T}$, Yamashita $\mathrm{H}$, Muramatsu M, Kobayashi S and Iwase H. Clinical value of the wild-type estrogen receptor beta expression in breast cancer. Cancer letters. 2001; 163:207-212.

14. Omoto $\mathrm{Y}$, Kobayashi S, Inoue S, Ogawa S, Toyama T, Yamashita H, Muramatsu M, Gustafsson JA and Iwase H. Evaluation of oestrogen receptor beta wild-type and variant protein expression, and relationship with clinicopathological factors in breast cancers. European journal of cancer. 2002; 38:380-386.

15. Palmieri C, Lam EW, Mansi J, MacDonald C, Shousha S, Madden P, Omoto Y, Sunters A, Warner M, Gustafsson JA and Coombes RC. The expression of ER beta cx in human breast cancer and the relationship to endocrine therapy and survival. Clin Cancer Res. 2004; 10:2421-2428.

16. Chantzi NI, Tiniakos DG, Palaiologou M, Goutas N, Filippidis T, Vassilaros SD, Dhimolea E, Mitsiou DJ and Alexis MN. Estrogen receptor beta 2 is associated with poor prognosis in estrogen receptor alpha-negative breast carcinoma. Journal of cancer research and clinical oncology. 2013; 139:1489-1498.

17. Guo L, Zhu Q, Yilamu D, Jakulin A, Liu S and Liang T. Expression and prognostic value of estrogen receptor beta in breast cancer patients. International journal of clinical and experimental medicine. 2014; 7:3730-3736.

18. Qui WS, Yue L, Ding AP, Sun J, Yao Y, Shen Z and Fan LH. Co-expression of ER-beta and HER2 associated with poorer prognosis in primary breast cancer. Clinical and investigative medicine Medecine clinique et experimentale. 2009; 32:E250-260.

19. Shaaban AM, Green AR, Karthik S, Alizadeh Y, Hughes TA, Harkins L, Ellis IO, Robertson JF, Paish EC, Saunders PT, Groome NP and Speirs V. Nuclear and cytoplasmic expression of ERbeta1, ERbeta2, and ERbeta5 identifies distinct prognostic outcome for breast cancer patients. Clin Cancer Res. 2008; 14:5228-5235.

20. Sugiura H, Toyama T, Hara Y, Zhang Z, Kobayashi S, Fujii Y, Iwase H and Yamashita H. Expression of estrogen receptor beta wild-type and its variant ERbetacx/beta2 is correlated with better prognosis in breast cancer. Japanese journal of clinical oncology. 2007; 37:820-828.

21. Wen XF, Shen Z, Shen ZZ, Nguyen M and Shao ZM. The expression of ER beta protein correlates with vascular endothelial growth factor and its prognostic significance in human breast cancer. Oncology reports. 2002; 9:937-944.

22. Wimberly H, Han G, Pinnaduwage D, Murphy LC, Yang XR, Andrulis IL, Sherman M, Figueroa J and Rimm DL. ERbeta splice variant expression in four large cohorts of human breast cancer patient tumors. Breast cancer research and treatment. 2014; 146:657-667.

23. Yan M, Rayoo M, Takano EA, kConFab I and Fox SB. Nuclear and cytoplasmic expressions of ERbeta1 and ERbeta2 are predictive of response to therapy and alters prognosis in familial breast cancers. Breast cancer research and treatment. 2011; 126:395-405.

24. Markey GC, Cullen R, Diggin P, Hill AD, Mc Dermott EW, O'Higgins NJ and Duffy MJ. Estrogen receptor-beta mRNA is associated with adverse outcome in patients with breast cancer. Tumour Biol. 2009; 30:171-175.

25. Zhang H, Zhang Z, Xuan L, Zheng S, Guo L, Zhan Q, Qu X, Zhang B, Wang Y, Wang X and Song Y. Evaluation of ERalpha, ER-Beta1 and ER-Beta2 expression and correlation with clinicopathologic factors in invasive luminal subtype breast cancers. Clin Transl Oncol. 2012; 14:225-231.

26. Moore JT, McKee DD, Slentz-Kesler K, Moore LB, Jones SA, Horne EL, Su JL, Kliewer SA, Lehmann $\mathrm{JM}$ and Willson TM. Cloning and characterization of human estrogen receptor beta isoforms. Biochemical and biophysical research communications. 1998; 247:75-78.

27. Savinov AY, Remacle AG, Golubkov VS, Krajewska M, Kennedy S, Duffy MJ, Rozanov DV, Krajewski S and 
Strongin AY. Matrix metalloproteinase 26 proteolysis of the NH2-terminal domain of the estrogen receptor beta correlates with the survival of breast cancer patients. Cancer research. 2006; 66:2716-2724.

28. Eckel-Passow JE, Lohse CM, Sheinin Y, Crispen PL, Krco CJ and Kwon ED. Tissue microarrays: one size does not fit all. Diagnostic pathology. 2010; 5:48.

29. Fuqua SA, Schiff R, Parra I, Moore JT, Mohsin SK, Osborne CK, Clark GM and Allred DC. Estrogen receptor beta protein in human breast cancer: correlation with clinical tumor parameters. Cancer research. 2003; 63:24342439.

30. Rosa FE, Caldeira JR, Felipes J, Bertonha FB, Quevedo FC, Domingues MA, Moraes Neto FA and Rogatto SR. Evaluation of estrogen receptor alpha and beta and progesterone receptor expression and correlation with clinicopathologic factors and proliferative marker Ki-67 in breast cancers. Human pathology. 2008; 39:720-730.

31. Speirs V, Malone C, Walton DS, Kerin MJ and Atkin SL. Increased expression of estrogen receptor beta mRNA in tamoxifen-resistant breast cancer patients. Cancer research. 1999; 59:5421-5424.

32. Smith L, Coleman LJ, Cummings M, Satheesha S, Shaw SO, Speirs V and Hughes TA. Expression of oestrogen receptor beta isoforms is regulated by transcriptional and post-transcriptional mechanisms. The Biochemical journal. 2010; 429:283-290.

33. Charn TH, Liu ET, Chang EC, Lee YK, Katzenellenbogen JA and Katzenellenbogen BS. Genome-wide dynamics of chromatin binding of estrogen receptors alpha and beta: mutual restriction and competitive site selection. Molecular endocrinology. 2010; 24:47-59.

34. Fisher B, Anderson S, Bryant J, Margolese RG, Deutsch M, Fisher ER, Jeong JH and Wolmark N. Twenty-year followup of a randomized trial comparing total mastectomy, lumpectomy, and lumpectomy plus irradiation for the treatment of invasive breast cancer. The New England journal of medicine. 2002; 347:1233-1241.

35. Gourgou-Bourgade S, Cameron D, Poortmans P, Asselain B, Azria D, Cardoso F, A’Hern R, Bliss J, Bogaerts J, Bonnefoi H, Brain E, Cardoso MJ, Chibaudel B, et al. Guidelines for time-to-event end point definitions in breast cancer trials: results of the DATECAN initiative (Definition for the Assessment of Time-to-event Endpoints in CANcer trials)dagger. Ann Oncol. 2015; 26:873-879.

36. Mandusic V, Dimitrijevic B, Nikolic-Vukosavljevic D, Neskovic-Konstantinovic Z, Kanjer K and Hamann U. Different associations of estrogen receptor beta isoforms, ERbeta1 and ERbeta2, expression levels with tumor size and survival in early- and late-onset breast cancer. Cancer letters. 2012; 321:73-79.

37. Wu X, Subramaniam M, Negron V, Cicek M, Reynolds C, Lingle WL, Goetz MP, Ingle JN, Spelsberg TC and Hawse JR. Development, characterization, and applications of a novel estrogen receptor beta monoclonal antibody. Journal of cellular biochemistry. 2012; 113:711-723.

38. Tierney JF, Stewart LA, Ghersi D, Burdett S and Sydes MR. Practical methods for incorporating summary time-toevent data into meta-analysis. Trials. 2007; 8:16.

39. Eliasen M, Gronkjaer M, Skov-Ettrup LS, Mikkelsen SS, Becker U, Tolstrup JS and Flensborg-Madsen T. Preoperative alcohol consumption and postoperative complications: a systematic review and meta-analysis. Annals of surgery. 2013; 258:930-942.

40. Liberati A, Altman DG, Tetzlaff J, Mulrow C, Gotzsche PC, Ioannidis JP, Clarke M, Devereaux PJ, Kleijnen J and Moher D. The PRISMA statement for reporting systematic reviews and meta-analyses of studies that evaluate healthcare interventions: explanation and elaboration. Bmj. 2009; 339:b2700.

41. Stroup DF, Berlin JA, Morton SC, Olkin I, Williamson GD, Rennie D, Moher D, Becker BJ, Sipe TA and Thacker SB. Meta-analysis of observational studies in epidemiology: a proposal for reporting. Meta-analysis Of Observational Studies in Epidemiology (MOOSE) group. Jama. 2000; 283:2008-2012.

42. GA Wells BS, D O'Connell, J Peterson, V Welch, M Losos, P Tugwell,. (2015). The Newcastle-Ottawa Scale (NOS) for assessing the quality of nonrandomised studies in metaanalyses. (Canada: Ottawa Hospital Research Institute Website.).

43. Parmar MK, Torri V and Stewart L. Extracting summary statistics to perform meta-analyses of the published literature for survival endpoints. Statistics in medicine. 1998; 17:2815-2834. 